\title{
IOT Based Residential Security System using Arduino
}

\author{
Mr. Vilas C. Rathod ${ }^{1}$, Asmita Lodhavat ${ }^{2}$, Shradha Narwadkar ${ }^{3}$, Sahil Gore ${ }^{4}$, Pratik Kadam ${ }^{5}$ \\ Professor, Information Technology Department, MIT Polytechnic, Pune, Maharashtra, India ${ }^{1}$ \\ Diploma Student, Information Technology Department, MIT Polytechnic, Pune, Maharashtra, India ${ }^{2,3,4,5}$
}

\begin{abstract}
IoT refers to the infrastructure of connected physical devices which is growing at a rapid rate as huge number of devices and objects are getting associated to the Internet. Home security is a very useful application of IoT and we are using it to create an inexpensive security system for homes as well as industrial use. The system will inform the owner about any unauthorized entry or whenever the door is opened by sending a notification to the user. After the user gets the notification, he can take the necessary actions. The security system will use a microcontroller known as Arduino Uno to interface between the components, a magnetic Reed sensor to monitor the status, a buzzer for sounding the alarm, and a WiFi module, ESP8266 to connect and communicate using the Internet. The main advantages of such a system includes the ease of setting up, lower costs and low maintenance.
\end{abstract}

Keywords: Arduino Uno, ESP8266 (Wi-Fi Module), Reed Sensor Module, Bread board and Jump Wires

\section{INTRODUCTION}

IoT or Internet Things refers to the network of connected physical objects that can communicate and exchange data among themselves without the need of any human intervention. The paper is organized as follows: Section 1 discuss about the introduction of IOT and its applications. Section 2, gives a details review of the focus of the paper. Section 3 talks about the already existing systems in the same field. Section 4 proposed the working model of the proposed system, whereas section 5 gives information about the architecture of our systems and all the hardware used. Section 6 tells about the Blynk Application used in this system. Section 7 explains the experimental results followed by conclusion and future enhancement at Section 8 .

\section{LITERATURE REVIEW}

Design and Implementation of Security for Smart Home based on GSM technology was discussed by Govinda et al. (2014) that provides two methods to implement home security using IoT. One is using web cameras such that whenever there is any motion detected by the camera, it sounds an alarm and sends a mail to the owner. This method of detecting intrusion is quite good, albeit somewhat expensive due to the cost of the cameras involved in the process. The cameras need to be of good quality which means it should have a wide range and the picture quality should be high enough to detect movement. Also if you go for movable cameras such as dome cameras they will cost even more than the fixed ones.

\section{EXISTING SYSTEM}

SMS based system using GSM was proposed by Karri and Daniel (2005) propose to use internet services to send messages or alert to the house owner instead of the conventional SMS. Jayashri and Arvind (2013) have implemented a fingerprint based authentication system to unlock a door. This system helps users by only allowing the users whose fingerprint are authorized by the owner of the house. This system can also be used to monitor who all have used the sensor to gained entry into the house. The system is coupled with a few more home protection features such as gas leakage and fire accidents. Although a good system, fingerprint sensors are expensive and complex (as they need increased sensor resolution) to integrate into an IoT setup. Some experts also argue that only relying on a fingerprint sensor is not wise as it is relatively easy to lift someone's fingerprints and replicate them, which is why it is always advised to use fingerprint scanners in a two factor authentication systems where an additional layer of security is available in the form of PIN, passcode, voice recognition, etc. 
Vol. 8, Issue 3, March 2019

IV.

PROPOSE SYSTEM

Before we begin connecting the hardware, we have to get the ESP8266 set up by flashing the latest version of the firmware available for the module. This is because the chip comes with an older version of the AT command firmware pre-installed out of the box which cannot communicate with the Blynk libraries efficiently and will give an error with our code. To flash the latest firmware, download the ESP8266 flasher tool and the latest firmware from the internet which would be in the bin format and set up the ESP8266 to the Arduino Uno as described below in figure.

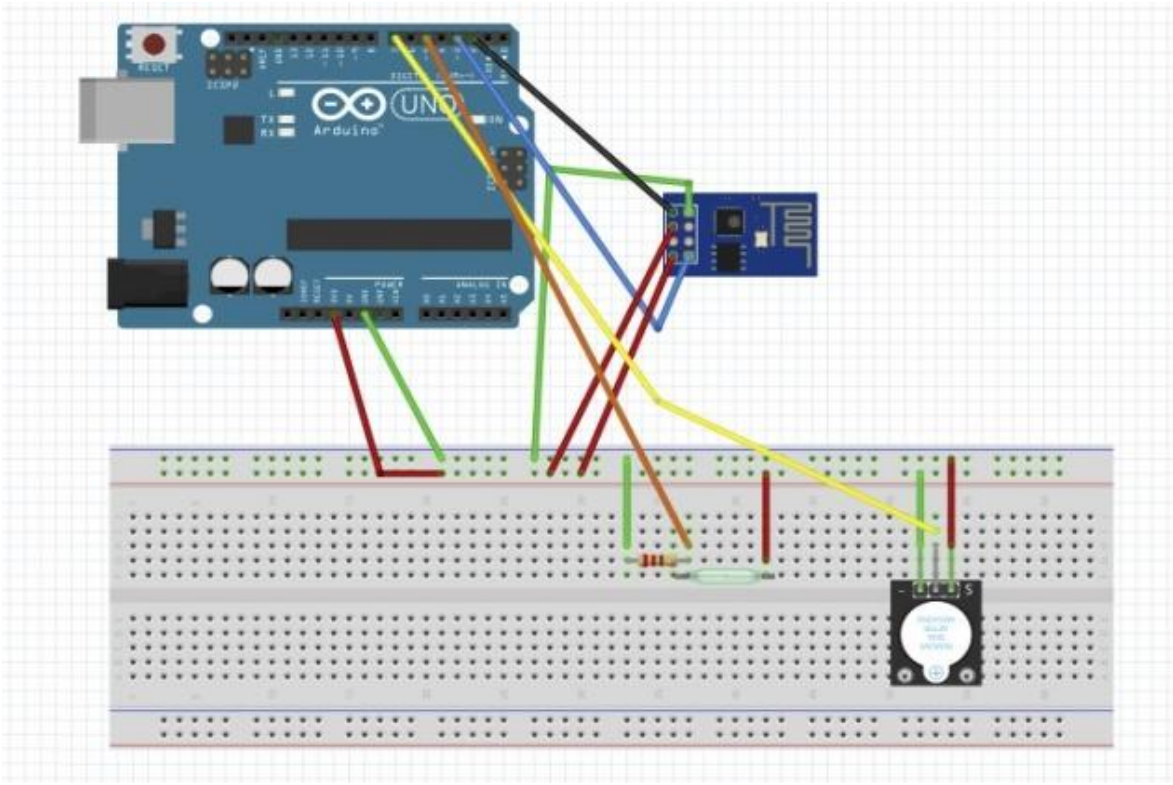

Once the ESP8266 has been flashed with the latest firmware, other components can be added to the configuration. For this we will need a breadboard to connect the microcontroller, reed sensor, buzzer and the ESP8266 using the jumper wires. The breadboard is used to interface between the various components available. It also makes it easy to connect multiple inputs to a single pin on the arduino board. Following sketch shown in figure 7 , which has been constructed using the Fritzing software shows how the components are supposed to be connected together using the breadboard and the jumper wires. The final configuration need not be identical to the given sketch, although the pins on each device needs to be connected to the same corresponding pins on the Arduino Uno board . The architecture diagram is shown in figure 8.

\section{SYSTEM ARCHITECTURES AND ITS COMPONENTS}

Various hardware materials are required to have a home automation system. Some of the essential components are listed below to have an idea about the proposed system

\subsection{Arduino Uno:}

Arduino is an open source, PC paraphernalia and programming organization, endeavor, and client group that plans and produce microcontroller packs for constructing programmed devices and intelligent object that can detect and control questions in the real world

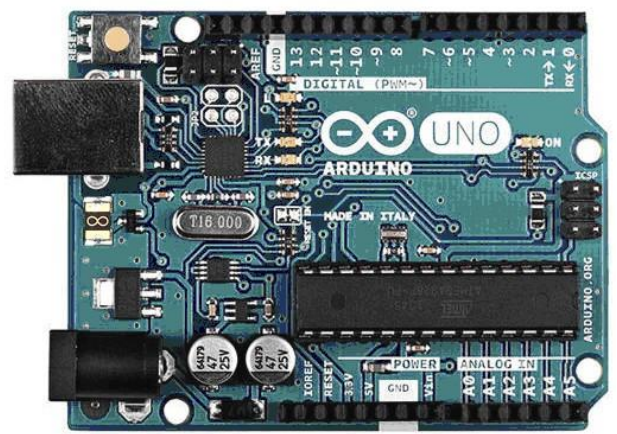

Figure : Arduino Uno. 


\subsection{ESP8266 (WiFi Module) :}

The ESP8266 is an ease Wi-Fi chip with full TCP/IP stack and MCU(Micro Controller unit) capacity created by Chinese. These are the primary arrangement of modules made with the ESP8266 by the outsider producer AI- Thinker and remain the most generally available. They are large alluded to as "ESP- xx modules

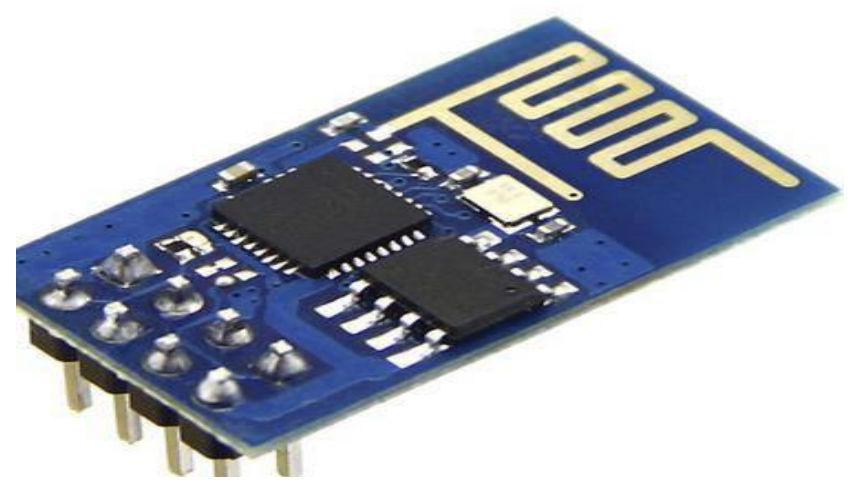

Figure : ESP8266 (Wi-Fi Serial Transceiver).

\subsection{Reed Sensor Module:}

In general, an electrical switch known as reed switch, worked by a connected field. It comprises of a fixed glass envelope where there are two ferrous flexible reeds and is loaded with idle gas called rhodium. At the point when an attractive substance ways to deal with the glass envelope, the reeds will meet up because of the attractive field subsequently finishing an electric circuit. At the point when the outer attractive field vanishes, two reeds will be isolated in view of their versatility, the circuit is likewise disconnected. It has been connected in printers, clothes washers, fridges, cameras, door magnets, window magnets, electromagnetic transfers, electronic measuring gadgets, level meters, gas meters, water meters, and so forth. Reed sensor module is shown in figure 3.

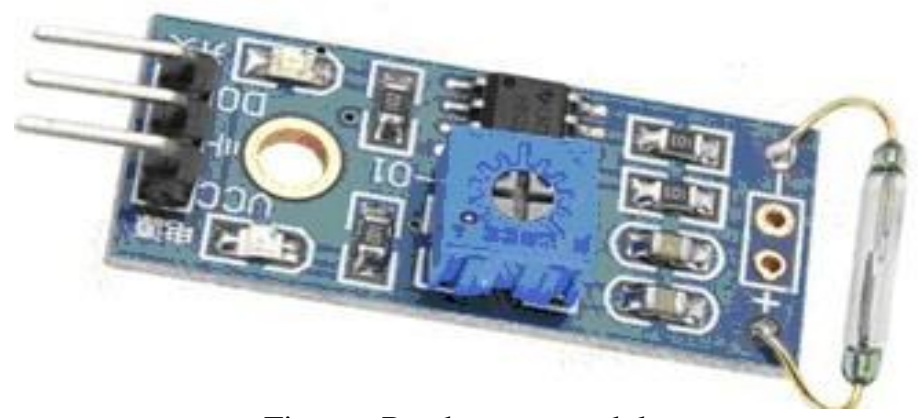

Figure : Reed sensor module

\subsection{Bread board and Jump wires:}

A breadboard is utilized to build and test circuits expeditiously afore finalizing any circuit design. The breadboard has many apertures into which route components like ICs and resistors can be connected. The apertures are generally spaced 0.1 " apart to put up standard DIP machinery. A typical breadboard that includes top and bottom power distribution rails is shown below figure 4. Jump wires are generally used to establish connectivity with bread board as shown in figure 5 .

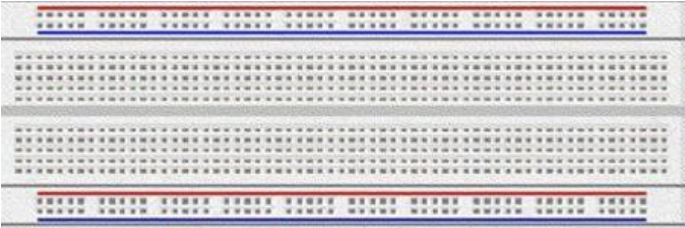

Figure : Bread board

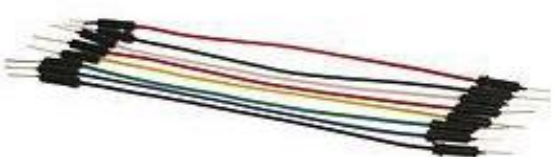

Figure : Jump Wires

\section{CONFIGURING BLYNK APP}

After the user installs the Blynk app on the smartphone, an account has to be created in the app to access its services. The first time the app is opened, it will ask to either sign in or create an account. Create an account and 
add a new project to get started as given in figure 9. Each project has its own authentication code which is used by the code to communicate with that particular model as provided in figure 10. To interface with our components, we need to add widgets to our model. To add widgets press ,,+" to add to the model. The app provides a neat interface to add all the required widgets and setting them up according to the code as shown in figure 11. The Blynk needs to be running in the background for the user to get real time notifications.

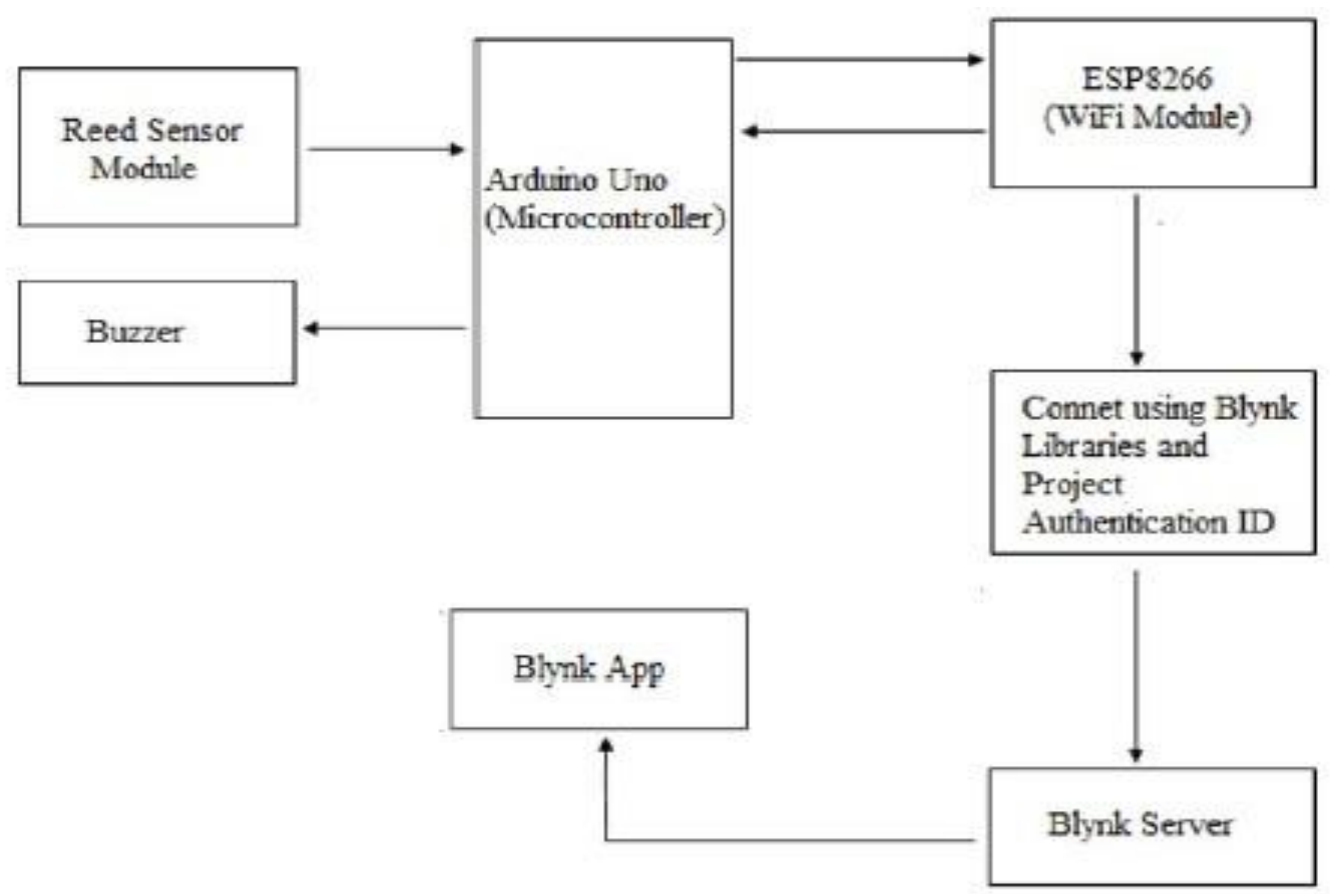

Figure : Configuration of Blynk App

VII. EXPERIMENTAL RESULTS

The experiment was carried out in Pentium iv $2.60 \mathrm{GHz}$ intel dual core processor, with 4 GB RAM, $15^{\text {ee }}$ LCD monitor with hard disk as $40 \mathrm{~GB}$. The software required are Blynk App, Arduino IDE, in windows operating system using $\mathrm{C}++$ programming language. The resultant system was checked thoroughly by repeating the motion of opening the door multiple times to see if each time a notification is sent or not and by remotely switching the buzzer on or off from the Blynk app which showed that the system works in the intended way and flawlessly. To test the endurance of the hardware, the setup was left turned on for a couple of hours and tested afterwards. The components got heated which is acceptable but still worked and the notification was shown in figure 12.

\section{CONCLUSION AND FUTURE SCOPE}

The sensors placed on the door informs the home owner as soon as the door is opened by sending a Push notification. The user will get this notification irrespective of whether the phone is locked or unlocked or even if any other app is opened at the moment. This was the main objective of the project, which is the user feels safe and not worry about any intrusion or break-ins when he is away from home. This setup can also be used in commercial offices where some areas are restricted for certain personnel, such a system will immediately inform the administrator of any unauthorized personnel trying to access such an area. Therefore the extensibility and applicability of such a system is only limited only by the imagination.

Another important component of the project is the connectivity between the ESP8266 (WiFi module) and the Blynk server. The system successfully connected to the Blynk server using the authentication token and the Blynk libraries. As a result, we were able to get the notification on our smart phones as soon as there was any change in the status of the reed module sensor. Also the additional ability to control the alarm remotely is very beneficial and can be very useful in some unforeseen circumstances. It was also observed that the Blynk app worked smoothly and carried out all communication between the hardware and the app very accurately. 
Vol. 8, Issue 3, March 2019

\section{REFERENCES}

[1]. Govinda K and Sai Krishna Prasad K and Sai Ram Susheel 2014 Intrusion detection system for smart home using laser rays International Journal for Scientific Research \& Development (IJSRD) 2 176-78

[2]. Karri V and Daniel Lim J S 2005 Method and Device to Communicate via SMS after a Security Intrusion 1st International Conf. on Sensing Technology Palmerston North New Zealand 21-23

[3]. Jayashri B and Arvind S 2013 Design and Implementation of Security for Smart Home based on GSM technology International Journal of Smart Home 7 201-08

[4]. Sowjanya G and Nagaraju S 2016 Design and Implementation Of Door Access Control And Security System Based On Iot Inventive Computation Technologies (ICICT), International Conference on Inventive

[5]. Cristian C, Ursache A, Popa D O and Florin Pop 2016 Energy efficiency and robustness for IoT: building a smart home security system Faculty of Automatic Control and Computers University Politehnica of Bucharest, Bucharest, Romania 43

[6]. Lee C T, Shen T C, Lee W D and Weng K W 2016 A novel electronic lock using optical Morse code based on the Internet of Things Proceedings of the IEEE International Conference on Advanced Materials for Science and Engineering eds. Meen, Prior \& Lam

[7]. Pooja P, Mitesh P, Vishwa P and Vinit N 2016 Home Automation Using Internet of Things Imperial Journal of Interdisciplinary Research (IJIR) 2 648-51

[8]. Anitha A, Paul G and Kumari S 2016 A Cyber defence using Artificial Intelligence International Journal of Pharmacy and Technology 8 25352-57

[9]. Anitha A, Kalra S and Shrivastav 2016 A Cyber defence using artificial home automation system using IoT International Journal of Pharmacy and Technology 8 25358-64 